\title{
Influence of Molecular Shape on the Dielectric Constant of Polar Liquids
}

\begin{abstract}
F. Buckley and A. A. Maryott
The Onsager theory of the static dielectric constant of polar liquids has been modified to allow for the influence of molecular shape. Equations have been derived for both prolate and oblate spheroids in which the permanent dipole is directed along the axis of symmetry. These equations have been applied to a number of pure polar liquids, exclusive of the hydrogen bonded liquids, where reliable data were available over extended ranges of temperature. A satisfactory interpretation of the variation of dielectric constant with temperature is obtained provided the volume of the molecular cavity is maintained fixed and independent of temperature rather than allowed to expand in proportion to the molar volume as assumed by Onsager. The required molecular shapes are in general accord with the actual molecular geometries. The behavior of dilute solutions and mixtures with a nonpolar solvent are also satisfactorily correlated using the same shape parameters. It appears to be unnecessary to consider specific interactions between neighboring molecules in order to account for the static dielectric behavior of these systems.
\end{abstract}

\section{Introduction}

The Onsager $[1]^{1}$ theory has been notably successful in relating electric dipole moments as determined in the vapor phase with the "static" dielectric constants of polar liquids. Exceptions are liquids in which association occurs, usually through hydrogen bonding, as in water and the alcohols, or for which the dipole moment is variable, as in ethylene chloride. For a large number of the more normal liquids, such as ethers, ketones, nitriles, and alkyl and aryl halides, the calculated values of dipole moment fall with more or less equal frequency above and below the measured gas values. The Onsager relation thus represents a rather satisfactory average. However, the agreement between observed and calculated values is far from perfect and discrepancies of the order of 10 to 20 percent are relatively common.

Recent extensions of the dielectric theory of liquids have usually followed one of two patterns. In the one case, following Onsager, the environment surrounding and interacting with any given molecule is treated as a continuum having the macroscopic dielectric constant and the molecular model is altered to accommodate either optical anisotropy [2] or geometrical anisotropy $[3,4]$ or both $[5,6]$. A second, more generalized and flexible approach was introduced by Kirkwood [7] and extended by others [8, 9, 10]. Interactions between the molecule and its immediate surroundings are treated by the specific methods of statistical mechanics and the medium becomes a continuum only outside of this region. However, a rigorous application of this procedure to liquids is usually prevented by a lack of detailed knowledge of the liquid structure. Consequently, it has been customary in practice to make certain simplifying assumptions, of a nature comparable to those inherent in the Onsager development, in order to obtain a "normalized" relation that should apply in the absence of specific closerange interactions. Experimental departures from the idealized equation are then assumed to be indicative of such interactions or association [2, 10, 11]. If this interpretation were justified, dielectric measurements would provide a very useful tool for investigating structure in liquids since independent evidence regarding these interactions is ordinarily lacking.

The simplicity and explicit nature of the Onsager approach make it desirable to investigate its utility in more detail than has been done heretofore. In particular, it is of interest to see whether any reasonable and simple modification of Onsager's molecular model can account for the dielectric behavior of polar liquids when subjected to wide changes in temperature or when progressively diluted with a nonpolar solvent. Under these conditions the effect of molecular interactions, if important, should vary markedly.

In this paper equations are developed for both prolate and oblate spheroids in a manner

1 Figures in brackets indicate the literature references at the end of this paper. 
formally analogous to Onsager's treatment of the spherical case. A solution for the prolate spheroid comparable to that employed here has been published by Abbott and Bolton [3]. Analysis of a large body of data on the dielectric constants of pure liquids, mixtures, and dilute solutions, excluding hydrogen bonded substances, shows that deviations from the Onsager equation can be rather systematically interpreted in terms of the spheroidal models and that these shapes are at least in qualitative accord with the actual molecular geometries. For these systems it appears to be unnecessary, in general, to consider additional factors involving specific molecular interactions or dipole association.

\section{Modified Onsager Equation}

The Onsager molecular model consists of a polarizable point dipole having a moment $\vec{\mu}_{0}$ and an isotropic polarizability $\alpha$ located at the center of an otherwise empty spherical cavity of volume $v$. The cavity is surrounded by a continuum having the macroscopic dielectric constant $\epsilon$. The field inside the cavity is conveniently separated into two parts, a reaction field $\vec{R}$ that is parallel to the instantaneous dipole axis and a cavity field $\vec{E}_{c}$ parallel to the externally applied field $\vec{E}$. In the absence of any applied field, the polarizable dipole is en-

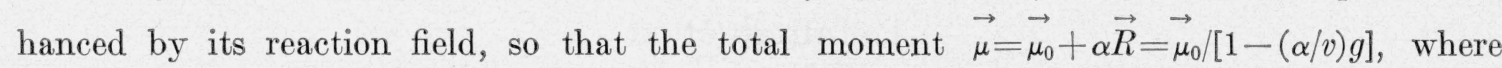
$R=g \mu / v$ and $g=(4 \pi / 3)(2 \epsilon-2) /(2 \epsilon+1)$. The cavity field, $\vec{E}_{c}=3 \epsilon /(2 \epsilon+1) \vec{E}$, both orients the permanent dipole and induces a moment that, when enhanced by its own reaction field, becomes $\vec{m}_{i}=\alpha \vec{E}_{c} /[1-(\alpha / v) g]$. The average total moment in the direction of $\vec{E}$ is then $\bar{m}=$ $m_{i}+\mu \overline{\cos \theta}$, where $\theta$ is the angle between $\vec{\mu}$ and $\vec{E}$. This model leads to the following relation between the dielectric constant and the molecular quantities:

$$
\frac{(\epsilon-1)(2 \epsilon-1)}{9 \epsilon} V=\frac{4 \pi N}{3}\left[\frac{\alpha}{1-\frac{\alpha}{v} g}+\frac{\mu_{0}^{2}}{3 k T\left(1-\frac{\alpha}{v} g\right)^{2}}\right],
$$

where $k$ is Boltzmann's constant, $T$ the absolute temperature, $V$ the molar volume, and $N$ Avogadro's number. The left-hand side of this expression is often called the Onsager-Kirkwood polarization, $P$.

It is convenient to express differences between theory and experiment in terms of a deviation factor, $G$, defined as $\mu_{0(\text { calc. })}^{2} / \mu_{0 \text { (gas) }}^{2}$. A value of unity for $G$ indicates agreement between the dipole moment calculated from the dielectric constant of the liquid and the measured gas value. The values of $G$ will depend upon the choice of polarizability and the volume assumed for the cavity. Onsager chose the cavity volume to be the same as the average volume occupied by the molecule, i. e., $v=V / N$, since eq (1) then reduces to the ClausiusMossotti equation for a nonpolar liquid, a relation which, though not exact, is a good approximation in such cases. Although the polarizability should include a small "atomic" contribution as well as the optical part, the former is seldom known with any degree of accuracy for polar substances even from measurements on the vapor and will be neglected in the analyses to follow. Values of $G$ derived from eq (1), hereafter designated as $G_{\text {Onsager }}\left(G_{\text {On. }}\right)$, have been obtained by using the relations $4 \pi N \alpha / 3=R_{D}$ (molar refraction for the $N \mathrm{a}_{D}$ line), and

$$
\alpha / v=\frac{3 R_{D}}{4 \pi V}=\frac{3\left(n_{D}^{2}-1\right)}{4 \pi\left(n_{D}^{2}+2\right)} .
$$

If one alters the Onsager model only to the extent of replacing the spherical cavity by one of equal volume having the shape of either a prolate or an oblate spheroid with the permanent dipole directed along the axis of symmetry, one obtains an equation of the form

$$
\frac{(\epsilon-1)(2 \epsilon+1)}{9 \epsilon} V=\frac{4 \pi N}{3}\left[\frac{\alpha k_{\alpha}}{1-\frac{\alpha}{v} g k_{R}}+\frac{\mu_{0}^{2}}{3 k T\left(1-\frac{\alpha}{v} g k_{R}\right)^{2}}\right] k_{c} \text {. }
$$




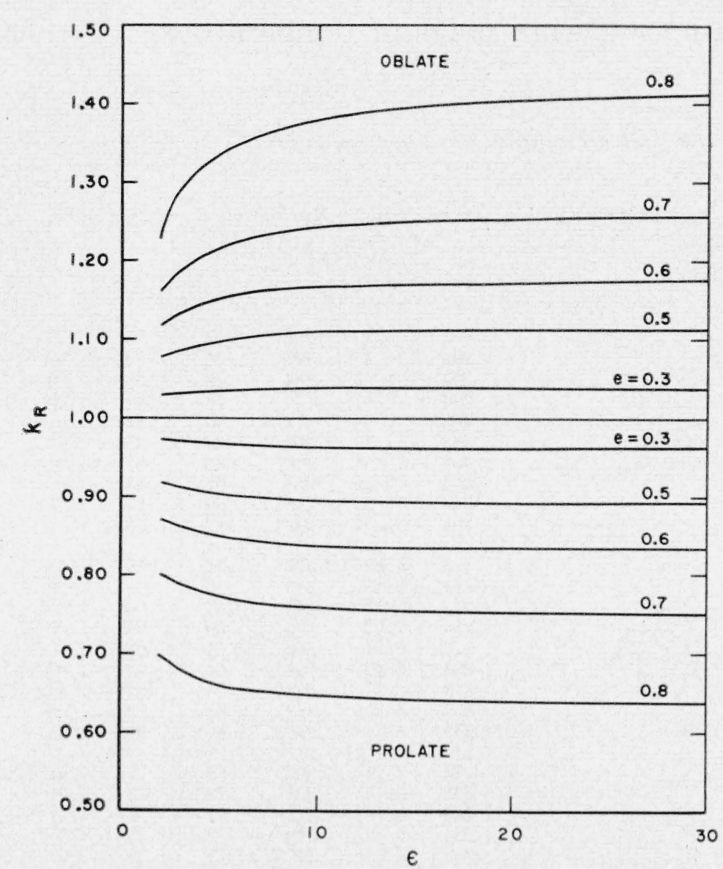

Figure 1. Reaction field correction coefficient as a function of dielectric constant for various values of molecular eccentricity.

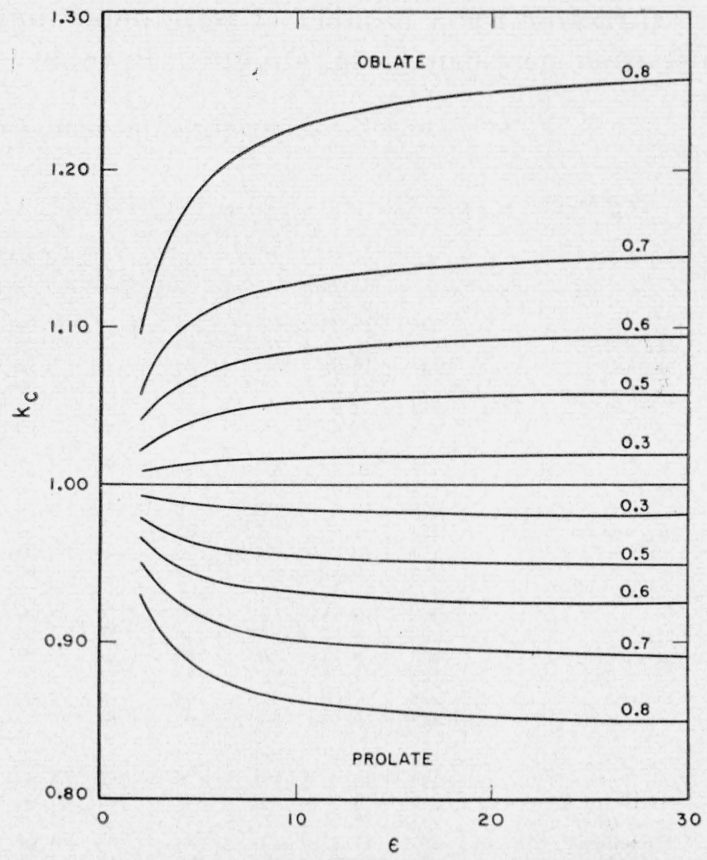

Figure 2. Cavity field correction coefficient as a function of dielectric constant for various values of molecular eccentricity.

The coefficients $k_{\alpha}, k_{R}$, and $k_{c}$ depend on the dielectric constant of the medium and the shape of the spheroid. $k_{R}$ is the ratio of the reaction field of the spheroid to that of the equivalent sphere, and $k_{c}$ is the corresponding ratio for the cavity fields. For the sphere, $k_{\alpha}=k_{R}=k_{c}=1$, and eq (2) reduces to Onsager's equation. Details of the mathematical development, as well as the explicit functions defining $k_{R}, k_{c}$, and $k_{\alpha}$, are found in the appendix. To facilitate application of eq (2), it has been assumed that the product $k_{\alpha} k_{c}=1$. This approximation is adequate for the present purpose because $k_{\alpha} k_{c}$ is generally close to unity and practically independent of temperature.

Figure 1 shows the variation in reaction-field coefficient $k_{R}$ with dielectric constant for both prolate and oblate spheroids of various eccentricities $e$. A similar plot of the cavityfield coefficient $k_{c}$ is given in figure 2. For prolate spheroids, where $k_{R}$ and $k_{c}$ are both less than unity, the Onsager relation should give $G$ factors less than unity. In like manner, oblate spheroids should lead to values of $G_{0 n}$. greater than unity because both $k_{R}$ and $k_{c}$ are greater than unity.

\section{Analysis of Data on Pure Liquids}

Initial attempts to interpret deviations from Onsager's equation in terms of eq (2) for a number of pure polar liquids over rather extended ranges of temperature were only partially successful. In particular, $G$ factors adjusted to unity at some temperature by suitable choice of shape and eccentricity did not remain substantially independent of temperature. These variations were not systematically correlated with any simple picture of dipole association. The difficulty appeared to be inherent in Onsager's assumption requiring the cavity volume to vary with the thermal expansion of the liquid and was largely overcome when the cavity volume was allowed to remain independent of temperature. Moreover, analyses made by the authors on a number of nonpolar liquids, as well as the published work of others [12], show that the fit of eq (1) is improved if this volume is maintained constant. Although the empirical evidence for a constant cavity volume is sufficiently convincing, it should be emphasized that in the electrostatic theory this equivalent volume is determined by the electrostatic properties of the molecule and might well be essentially independent of the temperature. 
$G$ factors for a number of pure polar liquids over a wide range of temperature, together with other pertinent data, are given in table 1 .

TABLE 1. Summary of the analysis of the dielectric data for pure liquids ${ }^{a}$

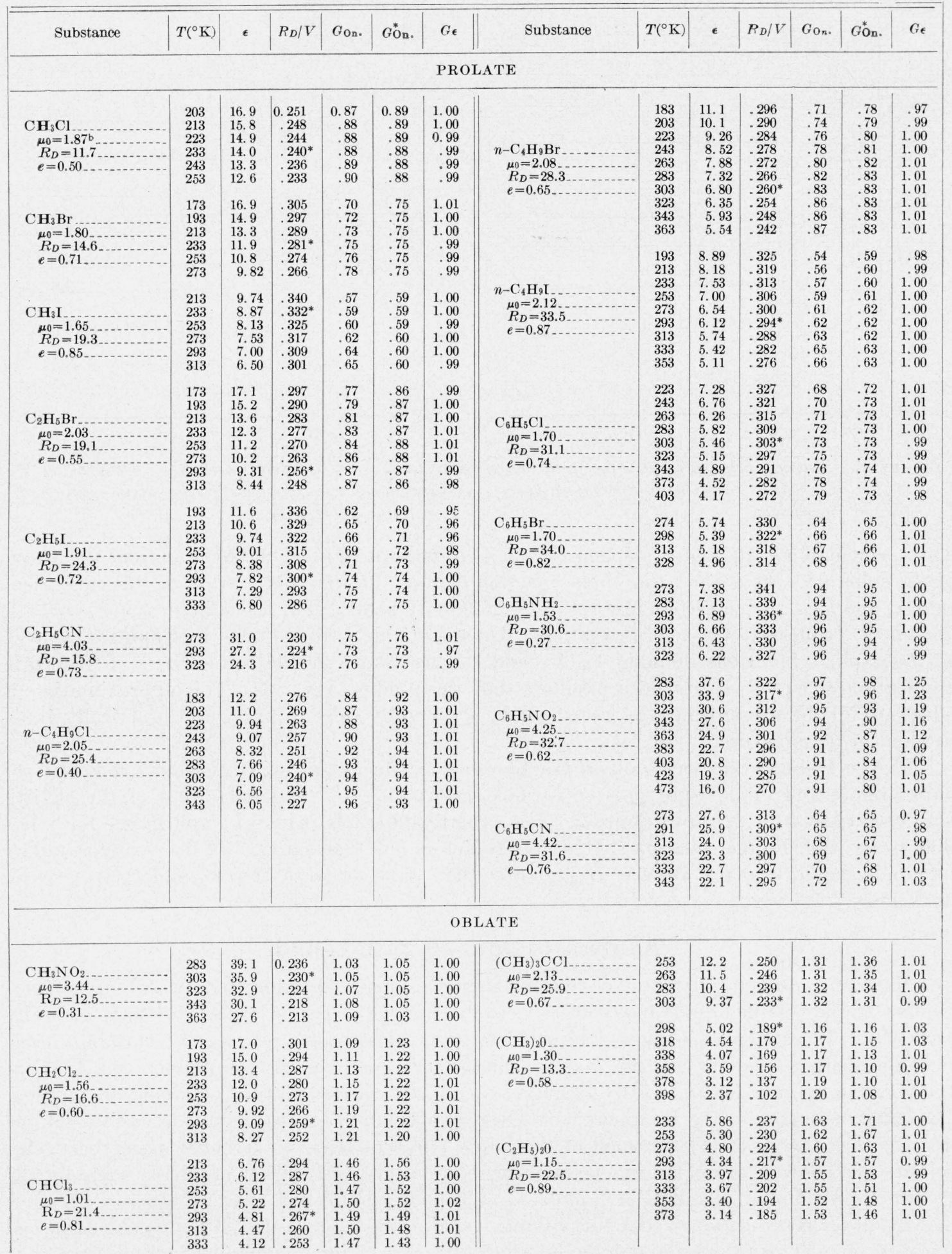


TABLE 1. Summary of the analysis of the dielectric data for pure liquids ${ }^{\text {_-Continued }}$

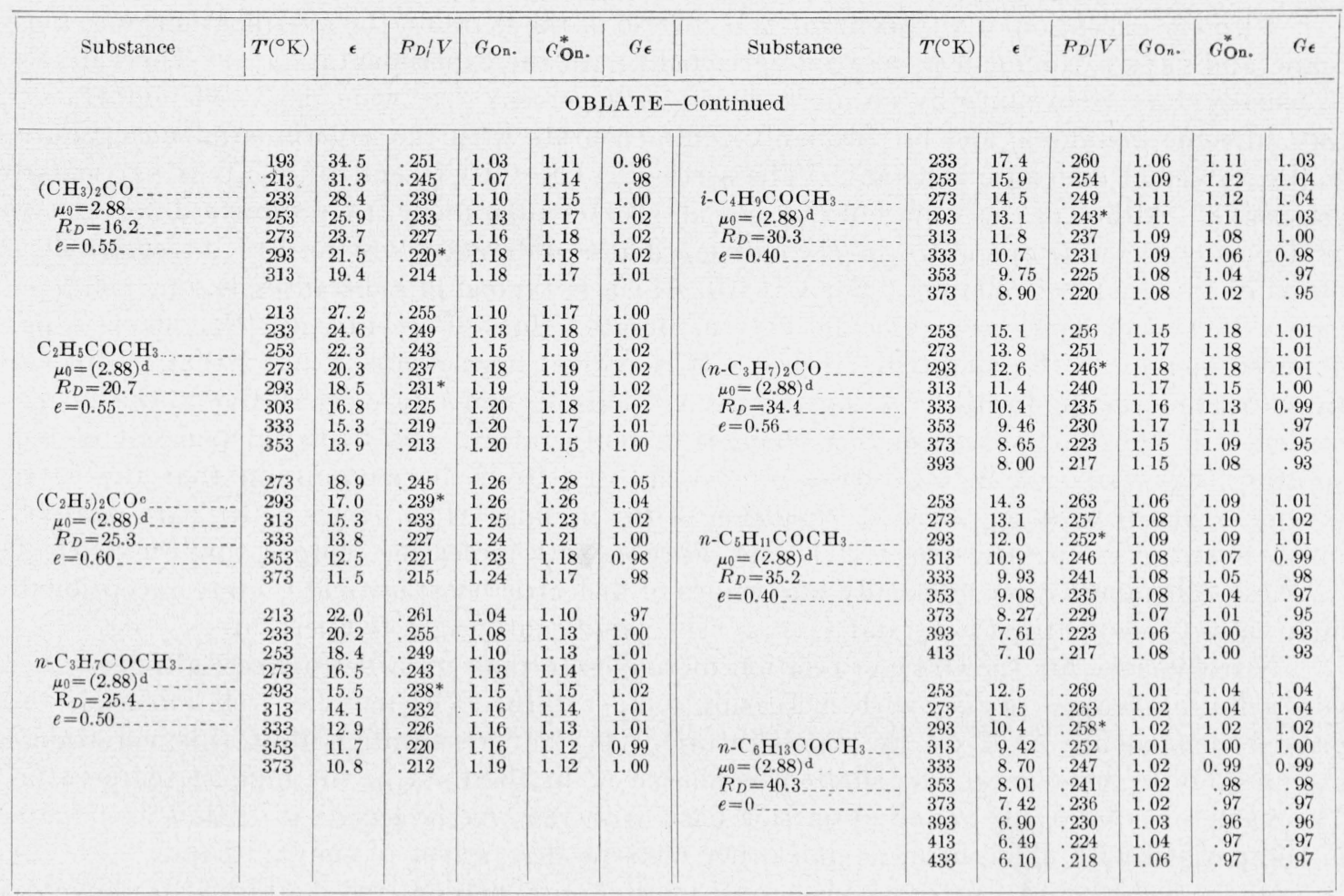

a Sources of data: A. A. Maryott and E. R. Smith, Table of dielectric constants of pure liquids, NBS Circular 514 (1951); A. A. Maryott, and Floyd Buckley, Table of dielectric constants and electric dipole moments of substances in the gaseous state, NBS Circular 537 (1953).

b All values of dipole moment given in Debye units ( 1 Debye $=10^{-18} \mathrm{esu}$ ).

-Data at the lower temperatures have been omitted since the tabulated values of dielectric constant are inconsistent with the graphical representation (R. N. Cole, J. Chem. Phys. 9, 251 (1951)).

d Value assumed to be the same as for acetone.

The factors designated as $G_{\mathrm{On}}^{*}$. and $G_{e}$ were obtained from eq (1) and (2), respectively, with the assumption of a fixed cavity volume. In these cases, the value of $R_{D} / V$ noted by an asterisk, a value usually pertaining to room temperature or its vicinity, was rather arbitrarily used. Estimates of the parameter $\alpha / v$ are not critical, and values that differ reasonably from the selected values do not entail significant changes in the results of the calculations or the interpretation of the data. Compounds have been classified as prolate or oblate, depending upon whether $G_{\mathrm{On}}^{*}$. is less or greater than unity. Using the reaction and cavity fleld coefficients appropriate to this shape, values of eccentricity were found that fitted eq (2). As the corresponding deviation factors $G_{e}$ have been adjusted near unity, they are only indicative with respect to their constancy with temperature and with respect to any correlation between the required cavity shape and the molecular geometries of the simpler types of molecules considered.

Table 1 shows that the values of $G_{\text {On. }}$ frequently increase with rising temperature. Such behavior, if confined to cases where $G_{\text {On. }}$ is less than unity, might be rather simply explained in terms of molecular interactions. Thus for molecules simulating prolate spheroids, dipole interactions would be such as to favor antiparallel alinement [13] and thereby tend to reduce the dipolar polarization. Value of $G_{\text {On. }}$. should then be less than unity but approach unity at more elevated temperatures as a consequence of the diminishing effect of such interactions. In like manner, values of $G_{\text {On. }}$ greater than unity, if interpretable in terms of parallel association in conformity with the general oblate geometry of this group, should also approach unity with rising temperature. However, except for ethyl ether, no such trend is evident. Furthermore, the values of $G^{*}$ on., calculated on the basis of a fixed cavity volume, generally show little or no variation with temperature for the prolate cases. Thus the evidence for association in these 
substances would appear to depend upon Onsager's assumption of an expanding cavity volume.

With the exception of nitrobenzene and several of the ketones, the modifications for cavity shape and cavity volume lead to good agreement with the experimental data. The values of $G_{e}$ usually vary from unity by no more than 1 or 2 percent over wide ranges of temperature, $180^{\circ}$ in some instances, and are probably commensurate with the experimental uncertainties in the values of dielectric constant. The series $\mathrm{CH}_{2} \mathrm{Cl}_{2}, \mathrm{CHCl}_{3}$, and $\left(\mathrm{C}_{2} \mathrm{H}_{5}\right)_{2} \mathrm{O}$ is particularly indicative. Although the values of $G_{\text {On }}$. are all greater than unity, they show a different type of dependence on temperature in each case. These differences are readily interpretable in terms of the modified relation. For $\mathrm{CH}_{2} \mathrm{Cl}_{2}$, which is typical of substances having relatively high values of dielectric constant and only moderate values of eccentricity, $G_{\text {On. }}$. shows a pronounced increase with rising temperature. It is evident from figures 1 and 2 that in this case the correction factors for the reaction and cavity fields, $k_{R}$ and $k_{\varepsilon}$, are insensitive to the changes in dielectric constant accompanying changes in temperature. As $G_{e}$ is independent of temperature, the variation in $G_{\text {On }}$. arises almost entirely from the assumption that the cavity volume expands with the thermal expansion of the liquids. The values of $G_{\text {Onn }}$. are relatively independent of temperature for $\mathrm{CHCl}_{3}$ and decrease with increasing temperature for $\left(\mathrm{C}_{2} \mathrm{H}_{5}\right)_{2} \mathrm{O}$. As these substances have unusually low values of dielectric constant and require exceptionally high values of eccentricity, $k_{R}$ and $k_{c}$ now vary significantly with temperature.

Nitrobenzene fits the Onsager relation more closely than most substances, although there is a definite decrease in $G_{\text {on }}$. with increasing temperature. The modified relation leads to a greater dependence of $G$ on the temperature. On the presumption that this variation is attributable to association, the shape was chosen to fit the data at the highest temperature. The value of eccentricity required on this basis is in reasonable accord with that anticipated in comparison with chlorobenzene and other benzene derivatives of similar shape.

Of the eight ketones listed only acetone, methyl ethyl ketone, and methly $n$-propyl ketone conform reasonably well to eq (1). Diethyl ketone and higher members of the series show a definite downward trend in $G_{e}$ with increasing temperature. This trend can hardly be attributed to association in view of the normal behavior of the lower members for which such association should be most pronounced. However, all of the ketones except acetone have flexible alkyl groups and it is not unreasonable to suppose that the effective shapes, especially of the higher members, may vary with the temperature. The observed trend in $G_{e}$ then suggests a change to a less oblate or more prolate shape as the temperature increases. Although similar complications might conceivably arise because of sufficient molecular flexibility in several other cases, namely, ethyl ether and the $n$-butyl halides, only the higher ketones appear to exhibit any abnormal behavior.

Table 2 contains a number of additional liquids for which reliable data were not available over a sufficient temperature range to be included in table 1 . The values of eccentricity required to fit eq (2) in the vicinity of room temperature are listed in the final column.

It is of interest to see how the shapes derived from the dielectric analyses correspond to estimates of the molecular shapes for those compounds where the dipole moment is directed along an axis of symmetry. In table 3 values of the axial ratio $b / a$ corresponding to the listed eccentricities are compared with those obtained by scaling models constructed from the FisherHirshfelder-Taylor atomic models. The dimension $a$ refers to the dipole axis while $b$ was taken as the largest dimension perpendicular to this axis. Some molecules containing flexible ethyl groups are included. For these cases, the values of $b$ were obtained from the most extended configuration and, consequently, these axial ratios represent upper limits. In no case do the two procedures lead to a definite conflict in the assignment of oblate and prolate character. However, the molecular geometry of $t$-butyl chloride is virtually spherical, whereas eq (2) requires a decidely oblate shape. A similar discrepancy, but in the reverse sense, is noted for methylene bromide. On the other hand, the high eccentricities needed for ethyl ether and triethylamine are close to those estimated for the most extended configurations. In the prolate cases the agreement is consistently good, except for iodobenzene. 
TABLE 2. Summary of the analysis of the dielectric data for pure liquids for which data over an extended temperature interval are not available :

\begin{tabular}{|c|c|c|c|c|c|c|c|}
\hline Substance & $\mu_{0}^{\mathrm{b}}$ & $R_{D}$ & $T$ & $\epsilon$ & $R_{D} / V$ & Gon. & $e$ \\
\hline \multicolumn{8}{|c|}{ PROLATE } \\
\hline $\begin{array}{l}\mathrm{CH}_{3} \mathrm{CN}_{n} \\
n-\mathrm{C}_{3} \mathrm{H}_{7} \mathrm{CN} \\
n-\mathrm{C}_{4} \mathrm{H}_{9} \mathrm{CN} \\
\mathrm{C}_{2} \mathrm{H}_{5} \mathrm{~N}_{2} \mathrm{O}_{2} \\
n-\mathrm{C}_{3} \mathrm{H}_{7} \mathrm{NO}_{2}\end{array}$ & $\begin{array}{l}3.97 \\
4.07 \\
4.12 \\
3.62 \\
3.66\end{array}$ & $\begin{array}{l}11.1 \\
20.4 \\
25.0 \\
17.0 \\
21.4\end{array}$ & $\begin{array}{l}293 . \\
293 \\
293 \\
303 \\
303\end{array}$ & $\begin{array}{l}37.5 \\
20.3 \\
17.4 \\
28.1 \\
23.2\end{array}$ & $\begin{array}{r}0.212 \\
.234 \\
.239 \\
.235 \\
.239\end{array}$ & $\begin{array}{l}0.80 \\
.60 \\
.58 \\
.96 \\
.95\end{array}$ & $\begin{array}{l}0.67 \\
.90 \\
.91_{5} \\
.30 \\
.30\end{array}$ \\
\hline $\begin{array}{l}n-\mathrm{C}_{3} \mathrm{H}_{7} \mathrm{I} \\
n-\mathrm{I}_{3} \mathrm{H}_{7} \mathrm{Br} \\
n-\mathrm{C}_{5} \mathrm{H}_{11} \mathrm{Br} \\
n-\mathrm{C}_{6} \mathrm{H}_{13} \mathrm{Br} \\
n-\mathrm{C}_{7} \mathrm{H}_{15} \mathrm{Br}\end{array}$ & $\begin{array}{c}2.04 \\
(2.10) \\
(2.10) \\
(2.10) \\
(2.10)\end{array}$ & $\begin{array}{l}28.9 \\
26.6 \\
33.1 \\
37.7 \\
42.1\end{array}$ & $\begin{array}{l}293 \\
298 \\
298 \\
298 \\
298\end{array}$ & $\begin{array}{l}\text { 7. } 00 \\
\text { 8. } 09 \\
\text { 6. } 31 \\
\text { 5. } 82 \\
\text { 5. } 33\end{array}$ & $\begin{array}{l}.297 \\
.259 \\
.265 \\
.266 \\
.267\end{array}$ & $\begin{array}{l}.68 \\
.84 \\
.82 \\
.83 \\
.81\end{array}$ & $\begin{array}{l}.80 \\
.60 \\
.65 \\
.65 \\
.67\end{array}$ \\
\hline $\begin{array}{l}n-\mathrm{C}_{8} \mathrm{H}_{19} \mathrm{Br} \\
n-\mathrm{C}_{9} \mathrm{H}_{19} \mathrm{Br} \\
n-\mathrm{C}_{10} \mathrm{H}_{21} \mathrm{Br}- \\
n-\mathrm{C}_{12} \mathrm{H}_{25} \mathrm{Br} \\
n-\mathrm{C}_{14} \mathrm{H}_{29} \mathrm{Br}\end{array}$ & $\begin{array}{r}(2.10) \\
(2.10) \\
(2.10) \\
(2.10) \\
(2.10)\end{array}$ & $\begin{array}{l}46.7 \\
51.5 \\
56.4 \\
66.1 \\
74.6\end{array}$ & $\begin{array}{l}298 \\
298 \\
298 \\
298 \\
298\end{array}$ & $\begin{array}{l}\text { 5. } 00 \\
\text { 4. } 74 \\
\text { 4. } 44 \\
\text { 4. } 07 \\
\text { 3. } 84\end{array}$ & $\begin{array}{l}.268 \\
.270 \\
.271 \\
.272 \\
.272\end{array}$ & $\begin{array}{l}.81 \\
.81 \\
.79 \\
.78 \\
.78\end{array}$ & $\begin{array}{l}.68 \\
.68 \\
.71 \\
.72 \\
.72\end{array}$ \\
\hline $\begin{array}{l}\mathrm{C}_{6} \mathrm{H}_{5} \mathrm{~F} \\
\mathrm{H}_{6} \mathrm{H}_{5} \mathrm{I}-\ldots \\
\mathrm{CH}_{2} \mathrm{Br}_{2}\end{array}$ & $\begin{array}{l}1.59 \\
1.70 \\
1.48\end{array}$ & $\begin{array}{l}26.0 \\
39.3 \\
21.9\end{array}$ & $\begin{array}{l}293 \\
293 \\
283\end{array}$ & $\begin{array}{l}\text { 5. } 47 \\
\text { 4. } 63 \\
7.77\end{array}$ & $\begin{array}{l}.277 \\
.353 \\
.320\end{array}$ & $\begin{array}{l}.83 \\
.45 \\
.99\end{array}$ & $0^{.65}$ \\
\hline \multicolumn{8}{|c|}{ OBLATE } \\
\hline $\begin{array}{l}\mathrm{CH}_{3} \mathrm{CHCl}_{2} \\
\mathrm{CH}_{3} \mathrm{CCl}_{3} \\
\left(\mathrm{CH}_{3}\right)_{2} \mathrm{CCl}_{2} \\
\mathrm{CH}_{3} \mathrm{COC}_{6} \mathrm{H}_{5} \\
\left(n-\mathrm{C}_{3} \mathrm{H}_{7}\right)_{2} \mathrm{O}_{2}\end{array}$ & $\begin{array}{l}\text { 2. } 06 \\
\text { 1. } 79 \\
2.27 \\
\text { 3. } 02 \\
1.21\end{array}$ & $\begin{array}{l}21.2 \\
26.2 \\
25.8 \\
34.5 \\
31.7\end{array}$ & $\begin{array}{l}291 \\
293 \\
293 \\
298 \\
299\end{array}$ & $\begin{array}{c}10.0 \\
7.52 \\
10.2 \\
17.4 \\
3.39\end{array}$ & $\begin{array}{r}0.252 \\
.263 \\
.248 \\
.294 \\
.230\end{array}$ & $\begin{array}{l}1.03 \\
1.12 \\
1.09 \\
1.12 \\
1.16\end{array}$ & $\begin{array}{r}0.30 \\
.50 \\
.45 \\
.45 \\
.65\end{array}$ \\
\hline $\begin{array}{l}\left(n-\mathrm{C}_{4} \mathrm{H}_{9}\right)_{2} \mathrm{O} \\
\left(\mathrm{C}_{2} \mathrm{H}_{5}\right)_{2} \mathrm{~S}_{2} \\
\left(\mathrm{C}_{2} \mathrm{H}_{5}\right)_{3} \mathrm{~N}\end{array}$ & $\begin{array}{l}1.17 \\
1.54 \\
0.66\end{array}$ & $\begin{array}{l}40.8 \\
28.5 \\
33.8\end{array}$ & $\begin{array}{l}298 \\
298 \\
298\end{array}$ & $\begin{array}{l}\text { 3. } 06 \\
\text { 5. } 72 \\
\text { 2. } 42\end{array}$ & $\begin{array}{l}.239 \\
.263 \\
.242\end{array}$ & $\begin{array}{l}1.15 \\
1.17 \\
1.31\end{array}$ & $\begin{array}{l}.62 \\
.58 \\
.80\end{array}$ \\
\hline
\end{tabular}

a Sources of data: A. A. Maryott and E. R. Smith, Table of dielectric constants of pure liquids, NBS Circular 514 (1951); A. A. Maryott and Floyd Buckley, Table of dielectric constants and electric dipole moments of substances in the gaseous state, NBS Circular 537 (1953).

b All values of dipole moment given in Debye units (1 Lebye $\left.=10^{-18} \mathrm{esu}\right)$.

TABLE 3.-Comparison of axial ratios determined from dielectric data and from molecular geometry

\begin{tabular}{|c|c|c|c|c|c|}
\hline Substance & $b / a$ (dielectric) & $b / a$ (geometry) & Substance & $b / a$ (dielectric) & $b / a$ (geometry) \\
\hline \multicolumn{3}{|c|}{ PROLATE } & \multicolumn{3}{|c|}{ OBLATE } \\
\hline $\begin{array}{l}\mathrm{CH}_{3} \mathrm{Cl} \\
\mathrm{CH}_{3} \mathrm{Br} \\
\mathrm{CH}_{3} \mathrm{I} \\
\mathrm{CH}_{3} \mathrm{CN} \\
\mathrm{C}_{6} \mathrm{H}_{5} \mathrm{NO} \mathrm{O}_{2} \\
\mathrm{C}_{6} \mathrm{H}_{5} \mathrm{Cl} \\
\mathrm{C}_{6} \mathrm{H}_{5} \mathrm{Br} \\
\mathrm{C}_{6} \mathrm{H}_{5} \mathrm{I} \\
\mathrm{C}_{6} \mathrm{H}_{5} \mathrm{CN}\end{array}$ & $\begin{array}{r}0.9 \\
.7 \\
.5 \\
.7 \\
.6 \\
.7 \\
.6 \\
.3 \\
.7\end{array}$ & $\begin{array}{r}0.8 \\
.7 \\
.7 \\
.7 \\
.8 \\
.8 \\
.8 \\
.7 \\
.7\end{array}$ & $\begin{array}{l}\mathrm{CH}_{2} \mathrm{Cl}_{2} \\
\mathrm{CH}_{2} \mathrm{Br}_{2} \\
\mathrm{CH} \mathrm{Cl}_{3} \\
\mathrm{CH}_{3} \mathrm{CCl}_{3} \\
\left(\mathrm{CH}_{3}\right)_{2} \mathrm{C} \mathrm{Cl}_{2} \\
\left(\mathrm{CH}_{3}\right)_{3} \mathrm{CCCl} \\
\left(\mathrm{CH}_{3}\right)_{2} \mathrm{O} \\
\left(\mathrm{C}_{2} \mathrm{H}_{5}\right)_{2} \mathrm{O} \\
\left(\mathrm{CH}_{3} \mathrm{C}_{2} \mathrm{CO}\right. \\
\left(\mathrm{C}_{2} \mathrm{H}_{5}\right)_{2} \mathrm{CO} \\
\left(\mathrm{C}_{2} \mathrm{H}_{5}\right)_{2} \mathrm{~S} \\
\left(\mathrm{C}_{2} \mathrm{H}_{5}{ }_{3} \mathrm{~N}\right. \\
\mathrm{CH}_{3} \mathrm{NO}_{2}\end{array}$ & $\begin{array}{l}1.3 \\
1.0 \\
1.7 \\
1.2 \\
1.1 \\
1.4 \\
1.2 \\
2.2 \\
1.2 \\
1.4 \\
1.2 \\
1.7 \\
1.0\end{array}$ & $\begin{array}{l}1.4 \\
1.7 \\
1.4 \\
1.0 \\
1.2 \\
1.0 \\
1.7 \\
2.0 \\
1.3 \\
1.8 \\
1.8 \\
1.8 \\
0.9\end{array}$ \\
\hline
\end{tabular}

Variations in the values of eccentricity obtained from the dielectric analysis are usually in the direction anticipated for members of homologous series and other related compounds. For example, the prolate eccentricity increases with increasing size of the halogen atom, $\mathrm{F}<\mathrm{Cl}<\mathrm{Br}<\mathrm{I}$, for the alkyl and aryl halides. The prolate eccentricity also increases with increasing chain length in homologous series of alkyl halides and nitriles, excepting certain first members of the series. 


\section{Analyses of Data on Solutions and Mixtures}

On the assumption that the Onsager-Kirkwood polarizations conform to the additive mixture law, eq (1) becomes for a two component mixture

$$
\begin{aligned}
P_{12}=\frac{\left(\epsilon_{12}-1\right)\left(2 \epsilon_{12}+1\right)}{9 \epsilon_{12}} V_{12}=f_{1} P_{1}+f_{2} P_{2}=\frac{4 \pi N}{3} f_{1} & {\left[\frac{\alpha_{1}}{1-\frac{\alpha_{1}}{v_{1}} g_{12}}+\frac{\mu_{1}^{2}}{3 k T\left(1-\frac{\alpha_{1}}{v_{1}} g_{12}\right)^{2}}\right] } \\
& +\frac{4 \pi N}{3} f_{2}\left[\frac{\alpha_{2}}{1-\frac{\alpha_{2}}{v_{2}} g_{12}}+\frac{\mu_{2}^{2}}{3 k T\left(1-\frac{\alpha_{2}}{v_{2}} g_{12}\right)^{2}}\right],
\end{aligned}
$$

where $f$ is the mole fraction, and $P_{1}$ and $P_{2}$ are the polarizations of the components in the mixture. The subscripts 1,2 , and 12 refer to the two components and the mixture, respectively. For dilute solutions of a polar solute in a nonpolar solvent, it is convenient to expand eq (3) in the Hedestrand [14] manner. The polarization of the solute at infinite dilution $P_{2}^{\infty}$ is then given by

$$
P_{2}^{\infty}=P_{1} \frac{M_{2}}{M_{1}}+P_{1}\left[\frac{2 \epsilon_{1}^{2}+1}{\epsilon_{1}\left(2 \epsilon_{1}+1\right)\left(\epsilon_{1}-1\right)}-\frac{8 \pi \frac{\alpha_{1}}{v_{1}}}{\left(1-\frac{\alpha_{1}}{v_{1}} g_{1}\right)\left(2 \epsilon_{1}+1\right)^{2}}\right]\left(\frac{\Delta \epsilon_{12}}{\Delta f_{2}}\right)_{f_{2} \rightarrow 0}-\frac{P_{1}}{d_{1}}\left(\frac{\Delta d_{12}}{\Delta f_{1}}\right)_{f_{2} \rightarrow 0},
$$

where $M$ is the molecular weight, $d$ the density, and

$$
P_{1}=\frac{\left(\epsilon_{1}-1\right)\left(2 \epsilon_{1}+1\right)}{9 \epsilon_{1}} \frac{M_{1}}{d_{1}}
$$

If the Clausius-Mossotti relation is accepted for the pure nonpolar solvent so that

$$
\alpha_{1} / v_{1}=\frac{3\left(\epsilon_{1}-1\right)}{4 \pi\left(\epsilon_{1}+2\right)}
$$

the second term in brackets simplifies to

$$
\frac{8 \pi \frac{\alpha_{1}}{v_{1}}}{\left(1-\frac{\alpha_{1}}{v_{1}} g_{1}\right)\left(2 \epsilon_{1}+1\right)^{2}}=\frac{6 P_{1} d_{1}}{M_{1}\left(2 \epsilon_{1}+1\right)^{2}}
$$

For spheroidal molecular shapes,

$$
P_{2}^{\infty}=\frac{4 \pi N}{3} \frac{\alpha_{2}}{1-\frac{\alpha_{2}}{v_{2}} g_{1} k_{R}}+\frac{\mu_{0}^{2} k_{c}}{3 k T\left(1-\frac{\alpha_{2}}{v_{2}} g_{1} k_{R}\right)^{2}}
$$

where the coefficients $k_{R}$ and $k_{c}$ are functions of the eccentricity of the solute and the dielectric constant of the solvent.

Data on six polar solutes in a number of nonpolar solvents having dielectric constants in the range 1.9 to 2.8 are given in table 4 . As in the case of the purə liquids, values of $G_{\text {On. }}$ fall below unity for $\mathrm{C}_{2} \mathrm{H}_{5} \mathrm{Br}, \mathrm{C}_{6} \mathrm{H}_{5} \mathrm{Br}, \mathrm{CH}_{3} \mathrm{CN}$, and $\mathrm{C}_{6} \mathrm{H}_{5} \mathrm{CN}$, and above unity for $\left(\mathrm{CH}_{3}\right)_{3} \mathrm{CCl}$ and $\mathrm{CHCl}_{3}$, indicating that these differences may be again correlated with molecular shape. When the data are interpreted in terms of the modified Onsager expression (eq 5), using, values of $k_{R}$ and $k_{C}$ corresponding to the eccentricities derived from the pure liquids, tha deviation factors $G_{e}$ are usually much closer to unity. The correction for shape usually leads to a value of the dipole moment within several percent of the measured gas value. Exceptions are $\mathrm{CH}_{3} \mathrm{CN}$ and $\mathrm{CHCl}_{3}$, where the corrections are decidedly too small. 

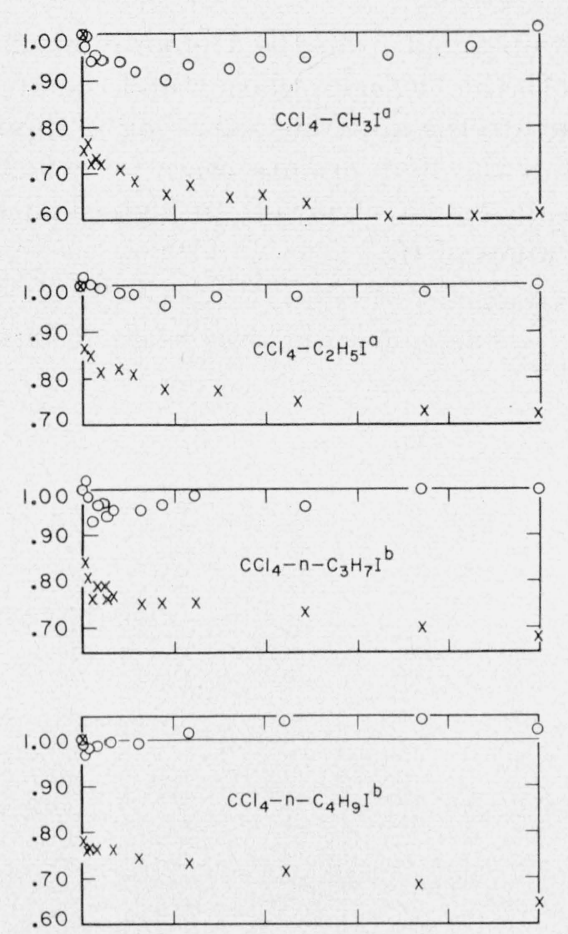

○
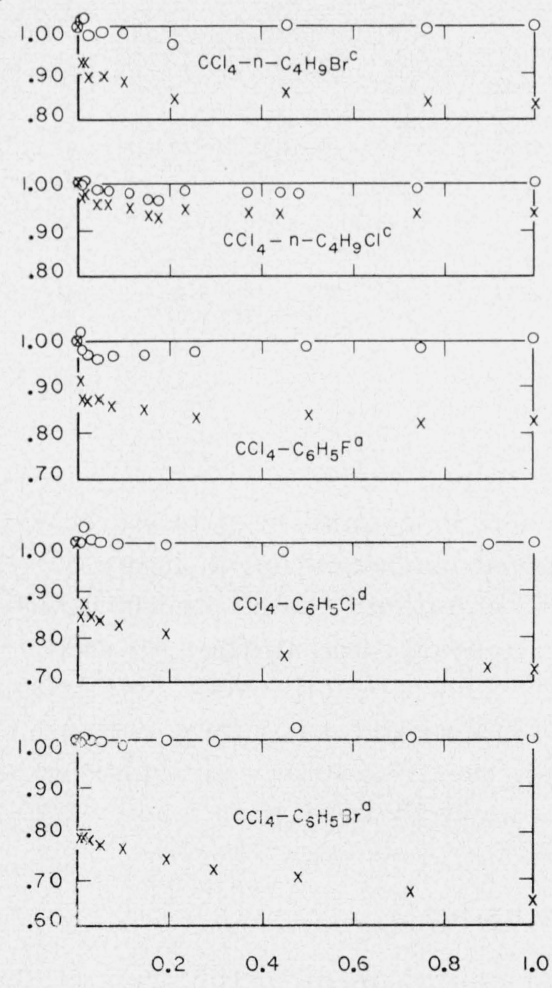
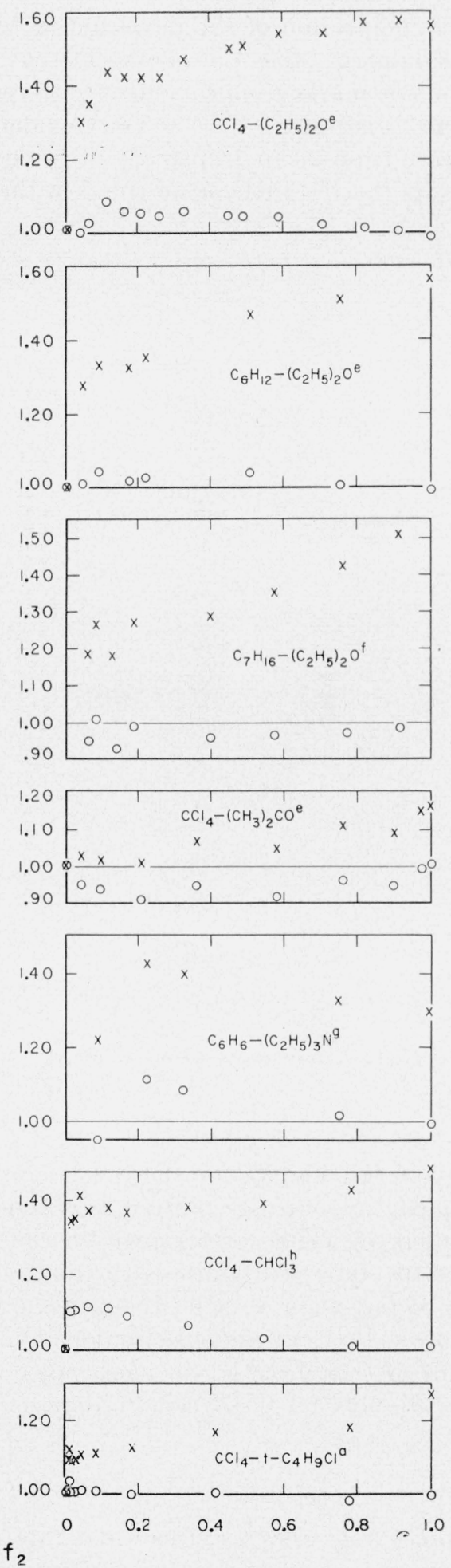

Figure 3. G-factor versus mole-fraction plots of mixture at $20^{\circ} \mathrm{C}$.

$\times, G_{\text {Onsager, }} \mathrm{O}, G_{e} ; f_{2}$, mole fraction of polar constituent.

a, A. Audsley and F. R. Goss, J. Chem. Soc. 1941, 864;, b, A. Audsley and F. R. Goss, J. Chem. Soc. 1942,358: c, A. Audsley and F. R. Goss, J. Chem. Soc. 1942, 497; d, F. R. Goss, J. Chem. Soc. 1937, 1918; e, D. P. Earp and S. Glasstone, J. Chem. Soc. 1935, $1709 ;$ f, G. Briegleb, Z. physik.Chem. B16, 249 (1932); g, D. D. Hammick, A. Norris, and L. E. Sutton, J. Chem. Soc. 1938, 1755; h, F. R. Goss, J. Chem. Soc. 1940, 755 . 
From a comparison of the deviation factors obtained from the Debye relation and listed in the last column of table 4, it is evident that both the Onsager relation and the present modification lead to a marked improvement with regard to the apparent variation in dipole moment from solvent to solvent. This is most evident in the four prolate cases for which values of $G_{\text {Debye }}$ decrease from 20 to 35 percent in going from media of lowest to highest dielectric constant, whereas there is little or no trend in the values of $G_{e}$.

TABLE 4. Hedestrand coefficients and $G$ factors for dilute solutions of several polar solutes in various nonpolar solvents

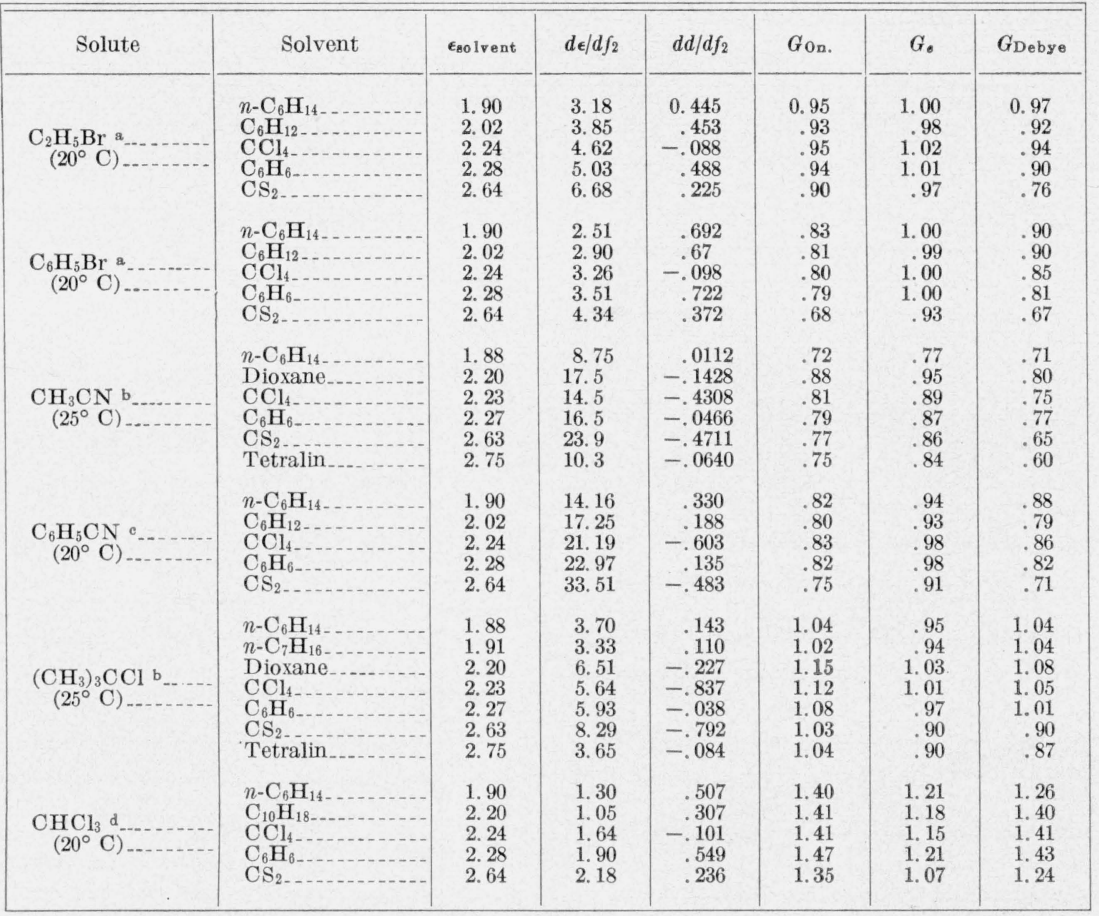

a E. G. Cowley and J. R. Partington, J. Chem, Soc. 1937, 130.

b J. W. Smith and L. B. Whitten, Trans. Faraday Soc. 47, 1304 (1951),

c E. G. Cowley and J. R. Partington, J. Chem. Soc. 1936, 1184.

d T. G. Scholte, Rec. trav. chim, 70, 50 (1951).

Values of $G_{\text {On, }}$ and $G_{e}$ for the polar constituent in a number of mixtures with $\mathrm{CCl}_{4}$ and other nonpolar solvents are plotted as a function of concentration in figure 3 . Slight differences between the values of $G$ shown for the pure liquid and those listed in tables 2 and 3 are due to slightly different values of the dielectric constant used in the two cases. In general, the values of $G_{\text {On. }}$ show a definite dependence on concentration, being closer to unity for the dilute solutions than for the pure liquid. On the other hand, the values of $G_{e}$ are substantially independent of concentration in most cases. For the 10 systems containing an alkyl or aryl halide in $\mathrm{CCl}_{4}$, only for $\mathrm{CH}_{3} \mathrm{I}$ does $G_{e}$ differs from unity by more than 4 percent over the entire concentration range.

\section{Conclusion}

The preceding analyses show that a decided improvement in the original Onsager treatment is obtained when the molecular cavity is altered to accommodate nonspherical molecular shapes, provided the volume of the cavity is kept independent of temperature. The behavior of pure polar liquids over an extended range of temperature and of dilute solutions and mixtures with a nonpolar solvent is, for the most part, satisfactorily correlated with a single set of molecular quantities involving size, shape, polarizability, and dipole moment. At the present time, however, limited knowledge concerning the appropriate values of polarizability, 
size, and shape would appear to preclude the determination of dipole moments from data on liquids and mixtures with an accuracy comparable with direct measurements on the vapor. In view of the rather arbitrary assignment of cavity volume, inadequate allowance of atomic polarizability, neglect of optical anisotropy, and other factors that could lead to an overly complicated molecular model, the exact values derived for the eccentricities are not of particular significance and should be regarded only as useful molecular parameters.

Liquids subject to the influence of hydrogen bonding have not been considered. They usually depart drastically from Onsager's theory and obviously require more specialized treatment as provided by the theory of Kirkwood. This need is also supported by a variety of independent evidence. On the other hand, much of the evidence supporting the concept of close-range structure or association in the systems under consideration results from a rather restricted interpretation of the dielectric behavior. Usually inherent is the assumption that, in the absence of association, the dipole moment enhanced by its reaction field would be the same as obtained from Onsager's model. With the modifications proposed here, it does not appear necessary, in general, to take into account specific molecular interactions and the approximation of a continuum for the molecular environment appears adequate.

\section{References}

[1] L. Onsager, J. Am. Chem. Soc. 58, 1486 (1936).

[2] J. N. Wilson, Chem. Rev. 25, 377 (1939).

[3] J. A. Abbott and H. C. Bolton, Trans. Faraday Soc. 48, 422 (1952).

[4] I. G. Ross and R. A. Sack, Proc. Phys. Soc. (London) [B] 63, 893 (1950).

[5] A. D. Buckingham, Australian J. Chem. 6, 93 (1953).

[6] T. G. Scholte, Physica 15, 437 and 450 (1949); Rec. trav. chim. 70, 50 (1951).

[7] J. G. Kirkwood, J. Chem. Phys, 7, 911 (1939).

[8] H. Frohlich, Theory of dielectries (Oxford University Press, London, 1949).

[9] W. F. Brown, Jr., J. Chem. Phys. 21, 1327 (1953).

[10] F. E. Harris and B. J. Alder, J. Chem. Phys. 21, 1031 (1953).

[11] G. Oster, J. Am. Chem. Soc. 68, 2036 (1946).

[12] C. J. F. Böttcher, Theory of electric polarization, ch. VII. (Elsevier Publishing Co., New York, N. Y., $1952)$.

[13] R. M. Fuoss, J. Am. Chem. Soc. 56, 1031 (1934).

[14] G. Hedestrand, Z. physik. Chem. B2, 428 (1929).

\section{Appendix}

It is required to find the reaction field, $\vec{R}_{\mu}$, of a point polarizable dipole located at the center of a cavity. The cavity is imbedded in an infinite homogeneous and continuous medium having a macroscopic dielectric constant of $\epsilon_{0}$.

The potential inside the cavity can be written

$$
\varphi_{i}^{(\mu)}=\varphi_{i}^{(0)}+\varphi_{i}^{(R)}
$$

where $\varphi_{i}^{(0)}$ reduces to the potential of a dipole for points in the neighborhood of the origin and $\varphi^{(R)}$ remains finite at all points within the cavity. Then

$$
\vec{R}_{\mu}=-\nabla \varphi_{i}^{(R)}
$$

For the cases that follow it is convenient to consider the corresponding problem for a charge, $Q$, situated at a point on the axis of symmetry and then to calculate $\varphi,{ }^{(\mu)}$ and $\vec{R}_{\mu}$ by the relations

$$
\begin{gathered}
\varphi,^{\mu}=\lim _{Q \delta x \rightarrow \mu} \frac{\delta x}{\partial x}, \\
\vec{R}_{\mu}=-\nabla \varphi,{ }^{(R)}
\end{gathered}
$$




\subsection{Sphere}

If a charge $Q$ is located at the point $\left(x_{0}, 0,0\right)$ then the potentials inside and outside the sphere are

$$
\begin{gathered}
\varphi_{i}=Q \sum_{n=0}^{\infty} \frac{x_{0}^{n}}{r^{n+1}} P_{n}(\cos \theta)+\sum_{n=0}^{\infty} B_{n} r^{n} P_{n}(\cos \theta) \\
\varphi_{0}=\frac{Q}{\epsilon_{0}} \sum_{n=0}^{\infty} C_{n} \frac{x_{0}^{n}}{r^{n+1}} P_{n}(\cos \theta)
\end{gathered}
$$

where $(r, \theta, \varphi)$ are the spherical polar coordinates of a point $P$, and the $P_{n}$ 's are the Legendre polynomials.

The usual boundary conditions over the surface of the sphere of radius $r$ suffice to determine the coefficients $B_{n}$ and $\mathrm{C}_{n}$. Only the $B_{n}$ are of interest. Calculation gives

so that

$$
B_{n}=-Q\left(\epsilon_{j}-1\right) \frac{x_{0}^{n}}{r^{2 n+1}}(n+1) \frac{1}{\left[\epsilon_{0}(n+1)+n\right]},
$$

$$
\begin{gathered}
\varphi_{i}^{(R)}=\lim _{Q \delta x_{0} \rightarrow \mu} \delta x_{0}\left(\frac{\partial \varphi_{1}}{\partial x_{0}}\right)=\frac{-\mu}{r^{3}} \frac{2\left(\epsilon_{0}-1\right)}{\left(2 \epsilon_{0}+1\right)} x \\
\vec{R}_{\mu}^{(s)}=-\nabla \varphi_{i}^{(R)}=\frac{\vec{\mu}}{r^{3}} \frac{2\left(\epsilon_{0}-1\right)}{\left(2 \epsilon_{0}+1\right)}=\vec{\mu} g_{s} .
\end{gathered}
$$

\subsection{Prolate Spheroid}

It is convenient to use prolate spheroidal coordinates $(\xi, \eta, \varphi)$ for which

$$
\left.\begin{array}{ll}
x=c \xi \eta & \\
y=c \sqrt{\left(\xi^{2}-1\right)\left(1-\eta^{2}\right)} \cos \varphi & -1 \leqq \eta \leqq 1 \\
z=c \sqrt{\left(\xi^{2}-1\right)\left(1-\eta^{2}\right)} \sin \varphi & 0 \leqq \varphi \leqq 2 \pi
\end{array}\right\}
$$

If $D$ is the distance between a point $P^{\prime}$ on the axis of symmetry (between the foci) and the point $P$, Hobson's expansion for $D^{-1}$ reduces to

$$
\frac{1}{D}=\frac{1}{c} \sum_{n=0}^{\infty}(2 n+1) P_{n}(\eta) Q_{n}(\xi) P_{n}\left(\eta^{\prime}\right),
$$

in which $P_{n}, Q_{n}$ are the Legendre functions of the first and second kind.

The required potentials can be written in the form

$$
\begin{gathered}
\varphi_{i}=\frac{Q}{c} \sum_{k=0}^{\infty}(2 k+1) P_{k}(\eta) Q_{k}(\xi) P_{k}\left(\eta^{\prime}\right)+\sum_{k=0}^{\infty} \alpha_{k} P_{k}(\eta) P_{k}(\xi), \\
\varphi_{0}=\frac{1}{\epsilon_{0}} \sum_{k=0}^{\infty} \beta_{k} P_{k}(\eta) Q_{k}(\xi) .
\end{gathered}
$$

The boundary conditions on the spheroid

give

$$
\left.\begin{array}{rlrl}
\varphi_{i} & =\varphi_{0} & \xi & =\xi_{0} \\
\epsilon_{i} \frac{d \varphi_{i}}{d \xi} & =\epsilon_{\supset} \frac{d \varphi_{0}}{d \xi} & & \xi=\xi_{0}
\end{array}\right\} .
$$

$$
\alpha_{k}=\frac{Q}{c}\left(\epsilon_{0}-1\right)(2 k+1) \frac{Q_{k}\left(\xi_{0}\right) Q_{k}^{\prime}\left(\xi_{0}\right) P_{k}\left(\eta^{\prime}\right)}{Q_{k}\left(\xi_{0}\right) P_{k}^{\prime}\left(\xi_{0}\right)-\epsilon_{0} Q_{k}^{\prime}\left(\xi_{0}\right) P_{k}\left(\xi_{0}\right)},
$$

in which the prime on the function denotes differentiation. 
The first term in the potential of the reaction field of a dipole at the origin reduces to

$$
\varphi_{R}^{(1)}=\frac{3 \mu}{c^{2}}\left(\epsilon_{0}-1\right) \frac{Q_{1}\left(\xi_{0}\right) Q_{1}^{\prime}\left(\xi_{0}\right) P_{1}(\xi) P_{1}(\eta)}{Q_{1}\left(\xi_{0}\right) P_{1}^{\prime}\left(\xi_{0}\right)-\epsilon_{0} Q_{1}^{\prime}\left(\xi_{0}\right) P_{1}\left(\xi_{0}\right)}
$$

and to the same approximation the reaction field of the dipole is

$$
\vec{R}_{\mu}^{(1)}=\frac{3 \mu}{c^{3}} \frac{\overrightarrow{\left(\epsilon_{0}-1\right)}}{\epsilon_{0}} \frac{\left[-1+\frac{1}{2 e} \log \frac{1+e}{1-e}\right]\left[\frac{-e}{1-e^{2}}+\frac{1}{2} \log \frac{1+e}{1-e}\right]}{\left[\frac{-e^{2}}{1-e^{2}}+\frac{\epsilon_{0}-1}{\epsilon_{0}}\left(-1+\frac{1}{2 e} \log \frac{1+e}{1-e}\right)\right]}=\mu g_{\|},
$$

where the eccentricity $e=\xi_{0}^{-1}=\sqrt{1-(b / a)^{2}}$ and $b / a$ is the axial radio of the spheroid.

The reaction field coefficient previously defined becomes

$$
k_{R}=\frac{R_{\mu}^{(1)}}{R_{\mu}^{(s)}}=\frac{3}{2} \frac{r^{3}}{c^{3}} \frac{2 \epsilon_{0}+1}{\epsilon_{0}} f(e) .
$$

The indefinite factor $r^{3} / c^{3}$ can be eliminated through the natural assumption that the volumes of the Onsager sphere and the spheroid are equal. This condition gives

so that finally

$$
\frac{r^{3}}{c^{3}}=\frac{1-e^{2}}{e^{3}}
$$

$$
k_{R}=\frac{3}{2} \frac{2 \epsilon_{0}+1}{\epsilon_{0}} \frac{1-e^{2}}{e^{3}} f(e) .
$$

A family of curves representing $k_{R}$ is shown in figure 1 .

The modified Onsager equation can be written

$$
\epsilon-1=4 \pi \frac{N}{V} \frac{\left(\vec{m} \cdot \vec{E}_{0}\right) \text { ave }}{E_{0}^{2}}=4 \pi \frac{N}{V E_{0}^{2}} \frac{\int e^{-\varepsilon / k T}\left(\vec{m} \cdot \vec{E}_{0}\right) d \omega}{\int e^{-\varepsilon / k T} d \omega}
$$

in which $\vec{E}_{0}, \vec{m}, N, V$ are the applied field, molecular moment, Avagadro's number, and molar volume, respectively. An extension of Onsager's treatment of the sphere gives, to a high approximation

$$
\begin{gathered}
\varepsilon=-\left(\overrightarrow{\mu \cdot \vec{E}_{\text {local }}}\right) \\
\vec{E}_{\text {local }}=\vec{\mu} \frac{g_{\|}}{1-\alpha_{\|} g_{\|}}+\frac{\vec{E}_{c \|}}{1-\alpha_{\|} g_{\|}}+\frac{\vec{E}_{c \perp}}{1-\alpha_{\perp} g_{\perp}}
\end{gathered}
$$

in which $\vec{E}_{c}$ is the cavity field, $\alpha$ is the polarizability, and $g=R / \mu$. The subscripts $\|$ and $\perp$ denote the directions parallel and perpendicular to the dipole axis. Hence, to a good approximation

in which

$$
\left(\frac{\vec{m} \cdot \vec{E}}{E_{0}^{2}}\right)_{\mathrm{ave}}=L\left\{\frac{1}{1-\alpha_{\|} g_{\|}}\left[\frac{1}{3} \alpha_{\|}+\frac{2}{3} \frac{M}{L} \alpha_{\perp}\left(\frac{1-\alpha_{\|} g_{\|}}{1-\alpha_{\perp} g_{\perp}}\right)\right]+\frac{\mu^{2}}{3 k T} \frac{1}{\left(1-\alpha_{\|} g_{\|}\right)^{2}}\right\}
$$

$$
\begin{array}{ll}
L=\frac{1}{1-\frac{a c^{2}}{2 \epsilon_{0}}\left(\epsilon_{0}-1\right) A_{x}} \quad A_{x}=\int_{0}^{\infty} \frac{d s}{\left(s+b^{2}\right)\left(s+a^{2}\right)^{3 / 2}} \\
M=\frac{1}{1-\frac{a c^{2}}{2 \epsilon_{0}}\left(\epsilon_{0}-1\right) A_{y}} \quad A_{y}=\int_{0}^{\infty} \frac{d s}{\left(s+b^{2}\right)^{2}\left(s+a^{2}\right)^{1 / 2}} .
\end{array}
$$


If the anisotropy of polarizability and shape are such that $\alpha_{\|} g_{\|}=\alpha_{\perp} g_{\perp}$ then

$$
\epsilon-1=\frac{4 \pi N}{3 V}\left\{\frac{\left(\frac{1}{3} \alpha_{\|}+\frac{2}{3} \frac{M}{L} \alpha_{\perp}\right)}{\left(1-\alpha_{\|} g_{\|}\right)}+\frac{\mu^{2}}{3 k T} \frac{1}{\left(1-\alpha_{\|} g_{\|}\right)^{2}}\right\} L
$$

and if the polarizability is isotropic then

$$
\epsilon-1=\frac{4 \pi N}{V}\left\{\frac{\alpha}{1-\alpha g_{\|}}\left(\frac{1}{3}+\frac{2}{3} \frac{M}{L} \frac{1-\alpha g_{\|}}{1-\alpha g_{\perp}}\right)+\frac{\mu^{2}}{3 k T} \frac{1}{\left(1-\alpha g_{\|}\right)^{2}}\right\} L .
$$

The determination of $g_{\perp}$ follows the method used to find $g_{\|}$. In the present case, the complete expansion of $D^{-1}$ is required. Hobson's expansion, for $P^{\prime}$ on the minor axis $(z)$, is

$$
\begin{aligned}
\frac{1}{D}=\frac{1}{c}\left\{\sum_{n=0}^{\infty}(2 n+1)\right. & P_{n}(\eta) Q_{n}(\xi) P_{n}(0) P_{n}\left(\xi^{\prime}\right) \\
& +2 \sum_{n=1}^{\infty} \sum_{m=1}^{n}(2 n+1)(-1)^{m}\left\{\frac{(n-m) !}{(n+m) !}\right\}^{2} P_{n}^{m}(\eta) Q_{n}^{m}(\xi) P_{n}^{m}(0) P_{n}^{m}\left(\xi^{\prime}\right) \cos m(\varphi-\pi / 2)
\end{aligned}
$$

in which $P_{n}^{m}(x), Q_{n}^{m}(x)$ are the Associated Legendre functions. The potentials are

$$
\begin{gathered}
\varphi_{i}^{(Q)}=Q \frac{1}{D}+\sum_{n=0}^{\infty} \alpha_{n} P_{n}(\eta) P_{n}(\xi)+\sum_{n=1}^{\infty} \sum_{m=1}^{n} \bar{\alpha}_{n m} P_{n}^{m}(\eta) P_{n}^{m}(\xi) \cos m(\varphi-\pi / 2) \\
\varphi_{0}^{(Q)}=\frac{Q}{\epsilon_{0}} \sum_{n=0}^{\infty} \beta_{n} P_{n}(\eta) Q_{n}(\xi)+\sum_{n=1}^{\infty} \sum_{m=1}^{n} \bar{\beta}_{n m} P_{n}^{m}(\eta) Q_{n}^{m}(\xi) \cos m(\varphi-\pi / 2) .
\end{gathered}
$$

The boundary conditions over the spheroid $\xi=\xi_{0}$ yield two sets of equations to determine $\left(\alpha_{n}, \beta_{n}\right)$ and $\left(\bar{\alpha}_{n m}, \bar{\beta}_{n m}\right)$. Only $\left(\alpha_{n}, \bar{\alpha}_{n m}\right)$ are of interest and algebraic solution gives

$$
\begin{gathered}
\alpha_{n}=\frac{Q}{c} 2(2 n+1)\left(\epsilon_{0}-1\right) \frac{Q_{n}\left(\xi_{0}\right) Q_{n}^{\prime}\left(\xi_{0}\right) P_{n}(0) P_{n}\left(\xi^{\prime}\right)}{Q_{n}\left(\xi_{0}\right) P_{n}^{\prime}\left(\xi_{0}\right)-\epsilon_{0} Q_{n}^{\prime}\left(\xi_{0}\right) P_{n}\left(\xi_{0}\right)} \cdot \\
\bar{\alpha}_{n m}=\frac{Q}{c} 2(2 n+1)(-1)^{m}\left\{\frac{(n-m) !}{(n+m) !}\right\}^{2}\left(\epsilon_{0}-1\right) \frac{Q_{n}^{m}\left(\xi_{0}\right) Q_{n}^{m^{\prime}}\left(\xi_{0}\right) P_{n}^{m^{\prime}}(0) P_{n}^{m}\left(\xi^{\prime}\right)}{Q_{n}^{m}\left(\xi_{0}\right) P_{n}^{m^{\prime}}\left(\xi_{0}\right)-\epsilon_{0} Q_{n}^{m^{\prime}}\left(\xi_{0}\right) P_{n}^{m}\left(\xi_{0}\right)} \cdot
\end{gathered}
$$

The only terms contributing to the potential of the reaction field for a dipole at the origin are those containing the $\bar{\alpha}_{n m}$. Hence the leading term is

so that

$$
\varphi_{\mu_{11}}^{(R)}=\frac{3}{2} \frac{\mu}{c^{2}}\left(\epsilon_{0}-1\right) \frac{Q_{1}^{1}\left(\xi_{0}\right) Q_{1}^{1^{\prime}}\left(\xi_{0}\right) P_{1}^{1}(\eta) P_{1}^{1}(\xi) \sin \varphi}{\epsilon_{0} Q_{1}^{1^{\prime}}\left(\xi_{0}\right) P_{1}^{1}\left(\xi_{0}\right)-Q_{1}^{1}\left(\xi_{0}\right) P_{1}^{1^{\prime}\left(\xi_{0}\right)}}
$$

$$
\overrightarrow{R_{\mu}}=\frac{3}{2} \frac{\vec{\mu}}{c^{3}}\left(\epsilon_{0}-1\right) \frac{Q_{1}^{1}\left(\xi_{0}\right) Q_{1}^{1^{\prime}}\left(\xi_{0}\right)}{Q_{1}^{1}\left(\xi_{0}\right) P_{1}^{1^{\prime}}\left(\xi_{0}\right)-\epsilon_{0} Q_{1}^{1^{\prime}}\left(\xi_{0}\right) P_{1}^{1}\left(\xi_{0}\right)} .
$$

After algebraic reduction, the required factor becomes

$$
\begin{gathered}
\frac{g_{\perp}}{g_{\text {॥ }}}=\frac{1}{2} \frac{\left[1+\frac{2}{x-1}\right]\left[\frac{\left(\epsilon_{0}-1\right)}{\epsilon_{0}}-\frac{1}{x}\right]}{\left[\frac{\epsilon_{0}-1}{\epsilon_{0}}+\frac{2}{x-1}\right]}>1 \\
x=\left(\frac{1-e^{2}}{e^{2}}\right)\left(-1+\frac{1}{2 e} \log \frac{1+e}{1-e}\right) .
\end{gathered}
$$


The cavity field coefficient previously defined is

$$
\begin{gathered}
k_{c}=E_{c}^{(e l l)} / E_{c}^{(s)}=\frac{2 \epsilon+1}{3 \epsilon} L=\beta k_{R} \\
\beta=\frac{9}{2}\left(\frac{1-e^{2}}{e^{2}}\right)^{2}\left[-1+\frac{1}{2 e} \log \frac{1+e}{1-e}\right]\left[\frac{1}{\left(1-e^{2}\right)}-\frac{1}{2 e} \log \frac{1+e}{1-e}\right]
\end{gathered}
$$

A family of curves representing $k_{c}$ is given in figure 2 .

Calculation shows that for practical ranges of $\epsilon$ the quantity $1-\alpha g_{\|} / 1-\alpha g_{\perp}$ is only slightly dependent upon $\epsilon$ (and therefore upon the temperature) and except for high eccentricities and exceptional values of $\alpha / r^{3}$ differs from unity by less than 10 percent. Moreover, the quantity $\left(\frac{1}{3}+\frac{2}{3} M / L\right) k_{c}$ differs insignificantly from unity except for very large eccentricities, so that

$$
\alpha k_{\alpha} k_{c}=\alpha\left\{\left(\frac{1}{3}+\frac{2}{3} \frac{M}{L}\left[1+\frac{1-\alpha g_{\|}}{1-\alpha g_{\perp}}\right]\right\} k_{c} \cong \alpha\right.
$$

can be taken as virtually constant.

The final equation used in the numerical computations reduces to

$$
\epsilon-1=\frac{4 \pi}{3} \frac{N}{V}\left\{\frac{\alpha}{1-\alpha g^{\mu}}+\frac{\mu^{2}}{3 k T} \frac{k_{c}}{\left(1-\alpha g_{\|}\right)^{2}}\right\} \frac{3 \epsilon}{2 \epsilon+1} .
$$

\subsection{Oblate Spheroid}

The appropriate coordinates are the oblate spheroidal coordinates for which

$$
\left.\begin{array}{lc}
x=c \xi \eta \sin \varphi & \xi \geqq 1 \\
y=c \sqrt{\left(\xi^{2}-1\right)\left(1-\eta^{2}\right)} & -1 \leqq \eta \leqq 1 \\
z=c \xi \eta \cos \varphi & 0 \leqq \varphi \leqq 2 \pi
\end{array}\right\} .
$$

Hobson's expansion for $D^{-1}$, in which the point $P^{\prime}$ is on the $y$-axis, reduces to

$$
\frac{1}{D}=\frac{1}{c} \sum_{n=0}^{\infty}(2 n+1) P_{n}(\bar{x}) Q_{n}(i \bar{y}) P_{n}\left(i \bar{y}^{\prime}\right)
$$

in which $\bar{x}=\sqrt{1-\eta^{2}}$ and $\bar{y}=\sqrt{\xi^{2}-1}$.

The required potentials are

$$
\begin{gathered}
\varphi_{i}^{(Q)}=\frac{Q}{c} \sum_{k=0}^{\infty}(2 k+1) P_{k}(\bar{x}) Q_{k}(i \bar{y}) P_{k}\left(i \bar{y}^{\prime}\right)+\sum_{k=0}^{\infty} \alpha_{k} P_{k}(i \bar{y}) P_{k}(\bar{x}) \\
\varphi_{0}^{(Q)}=\frac{1}{\epsilon_{0}} \sum_{k=0}^{\infty} \beta_{k} Q_{k}(i \bar{y}) P_{k}(\bar{x}) .
\end{gathered}
$$

The boundary conditions over the spheroid $\xi=\xi_{0}$ determine the $\left(\alpha_{k}, \beta_{k}\right)$. Only terms containing $\alpha_{k}$ contribute to the potential $\varphi_{i}^{(R)}$. Algebraic solution gives

$$
\alpha_{k}=\frac{-Q}{c}\left(\epsilon_{0}-1\right)(2 k+1) \frac{Q_{k}\left(i \bar{y}_{0}\right) Q_{k}^{\prime}\left(i \bar{y}_{0}\right) P_{k}\left(i \bar{y}^{\prime}\right)}{\epsilon_{0} P_{k}\left(i \bar{y}_{0}\right) Q_{k}^{\prime}\left(i \bar{y}_{0}\right)-P_{k}^{\prime}\left(i \bar{y}_{0}\right) Q_{k}\left(i \bar{y}_{0}\right)}
$$

so that the first term in the potential of the reaction field of the dipole at the origin is

$$
\varphi_{R}^{(1)}=-\frac{3 \mu}{c^{2}}\left(\epsilon_{0}-1\right) Q_{1}\left(i \bar{y}_{0}\right) Q_{1}^{\prime}\left(i \bar{y}_{0}\right) P_{1}(i \bar{y}) P_{1}(\bar{x})
$$


The reaction field of the dipole to the same approximation is

$$
\vec{R}_{\mu}^{(1)}=\frac{3 \vec{\mu}}{c^{3}} \frac{\left(\epsilon_{0}-1\right)}{\epsilon_{0}} \frac{\left[-1+\sqrt{\left.\frac{1-e^{2}}{e} \tan ^{-1} \frac{e}{\sqrt{1-e^{2}}}\right]\left[e \sqrt{1-e^{2}}-\tan ^{-1} \frac{e}{\sqrt{1-e^{2}}}\right]}\right.}{\left[e^{2}+\frac{\epsilon_{0}-1}{\epsilon_{0}}\left(-1+\frac{\sqrt{1-e^{2}}}{e} \tan ^{-1} \frac{e}{\sqrt{1-e^{2}}}\right)\right]}
$$

in which $e=\xi_{0}^{-1}$.

The reaction field coefficient, on the assumption that the volume of the spheroid and equivalent Onsager sphere are equal, reduces to

$$
k_{R}=\frac{3}{2} \frac{2 \epsilon_{0}+1}{\epsilon_{0}} \frac{\sqrt{1-e^{2}}}{e^{3}} \frac{\left[-1+\frac{\sqrt{1-e^{2}}}{e} \tan ^{-1} \frac{e}{\sqrt{1-e^{2}}}\right]\left[e \sqrt{1-e^{2}}-\tan ^{-1} \frac{e}{\sqrt{1-e^{2}}}\right]}{\left[e^{2}+\frac{\epsilon_{0}-1}{\epsilon_{0}}\left(-1+\frac{\sqrt{1-e^{2}}}{e} \tan ^{-1} \frac{e}{\sqrt{1-e^{2}}}\right)\right]}
$$

The calculation of the quantity $\left(\vec{m} \cdot \vec{E}_{0}\right)$ ave parallels that carried out for the prolate case. In view of the results obtained for this model, it will be assumed that to a good approximation

in which

$$
\frac{\left(\vec{m} \cdot \vec{E}_{0}\right)_{\text {are }}}{E_{0}^{2}}=\left\{\frac{\alpha}{\left(1-\alpha g_{\|}\right)}\left(\frac{1}{3}+\frac{2}{3} \frac{L}{M}\right)+\frac{\mu^{2}}{3 k T} \frac{1}{\left(1-\alpha g_{\|}{ }^{2}\right.}\right\} M
$$

$$
\begin{aligned}
\frac{1}{L} & =1-\frac{a^{2} b}{2 \epsilon_{0}}\left(\epsilon_{j}-1\right) A_{x} & A_{x} & =\int_{0}^{\infty} \frac{d s}{\left(s+a^{2}\right)^{2}\left(s+b^{2}\right)^{1 / 2}} \\
\frac{1}{M} & =1-\frac{a^{2} b}{2 \epsilon_{0}}\left(\epsilon_{0}-1\right) A_{y} & A_{y} & =\int_{0}^{\infty} \frac{d s}{\left(s+a^{2}\right)\left(s+b^{2}\right)^{3 / 2}} .
\end{aligned}
$$

The cavity field coefficient is

$$
k_{c}=\beta k_{R}=\frac{2 \epsilon_{0}+1}{\epsilon_{0}} M,
$$

where

$$
\frac{1}{\beta}=\frac{9}{2} \frac{1}{e^{4}}\left[-1+\frac{\sqrt{1-e^{2}}}{e} \tan ^{-1} \frac{e}{\sqrt{1-e^{2}}}\right]\left[\left(1-e^{2}\right)-\frac{\sqrt{1-e^{2}}}{e} \tan ^{-1} \frac{e}{\sqrt{1-e^{2}}}\right] .
$$

A family of curves representing $k_{c}$ is given in figure 2 .

The complete equation now becomes

$$
\epsilon_{0}-1=4 \pi \frac{N}{V}\left\{\frac{\alpha\left(\frac{1}{3}+\frac{2}{3} L / M\right)}{\left(1-\alpha g_{\|}\right)}+\frac{\mu^{2}}{3 k T} \frac{1}{\left(1-\alpha g_{\|}\right)^{2}}\right\} k_{c} \frac{3 \epsilon_{0}}{2 \epsilon_{0}+1} .
$$

Calculation shows that $k_{c}\left(\frac{1}{3}+\frac{2}{3} L / M\right)$ is essentially unity for practical ranges of $\epsilon_{0}$ and $e$, so that the working equation simplifies to

$$
\left(\epsilon_{0}-1=4 \pi \frac{N}{V}\left\{\frac{\alpha}{\left(1-\alpha g_{\|}\right)}+\frac{\mu^{2}}{3 k T} \frac{k_{c}}{\left(1-\alpha g_{\|}\right)^{2}}\right\} \frac{3 \epsilon_{0}}{2 \epsilon_{0}+1} .\right.
$$

In the case of the prolate spheroid, for which the calculations have been made, it should be noted that the inclusion of the correction factor $1-\alpha g_{i} / 1-\alpha g_{\perp}$ in the optical term of the polarization in part nullifies the effect of the correction for shape and increases this term to a value more nearly that of the Onsager approximation for a sphere. Calculations for a number of specific examples indicates that if this correction is made, the values of $G_{e}$ listed in the tables will be reduced by about 1 percent or less.

Washington, February 19, 1954. 